\title{
The Effect of Breathing Cycles Program on Heart Rate and Sleep Parameters in Healthy Young Adults
}

\author{
Jirawat Wattanapanyawech, PhD, Premtip Thaveeratitham, PT, PhD \\ Department of Physical Therapy, Faculty of Allied Health Sciences, Chulalongkorn University, Thailand
}

Received: August 3, 2020

Revised: October 6, 2020

Accepted: October 24, 2020

\section{Correspondence}

Premtip Thaveeratitham, PT, $\mathrm{PhD}$

Department of Physical Therapy,

Faculty of Allied Health Sciences,

Chulalongkorn University, Thailand

Tel +6622181591

E-mail Premtip.T@chula.ac.th

ORCID

Jirawat Wattanapanyawech

https://orcid.org/0000-0003-4358-4897

Premtip Thaveeratitham

https://orcid.org/0000-0002-6768-2363

(c) This is an Open Access article distributed under the terms of the Creative Commons Attribution Non-Commercial License (https://creativecommons.org/licenses/by-nc/4.0) which permits unrestricted non-commercial use, distribution, and reproduction in any medium, provided the original work is properly cited.
Background and Objective In recent years, sleep problems have been increasing globally, there exists an urgent need to develop a therapeutic program promoting sleep quality. This study investigated the effect of three types of deep breathing cycles programs on heart rate (HR) and sleep parameters in healthy young adults.

Methods Thirty healthy participants were recruited. HR and sleep parameters were measured via a smartwatch in all participants for two consecutive days. Participants were randomized into the forced deep breathing cycles (FDBC), non-forced deep breathing cycles (N-FDBC) and breathing control (BC) groups, with each participant performing the breathing cycle programs before sleep latency for 30 minutes.

Results N-FDBC showed a significant increase in sleep quality $(\mathrm{p}<0.001)$, time in bed $(\mathrm{p}<$ $0.05)$, and total sleep time ( $<0.001)$, as well as a significant decrease in sleep latency and \%awake during sleep $(\mathrm{p}<0.001)$. During sleep, maximum HR, minimum HR, average awake HR during sleep, average light sleep HR, and average deep sleep HR were significantly lower $(\mathrm{p}<0.01)$. For the FDBC group, significant increases in sleep latency $(p<0.05)$ and decreases in \%awake during sleep $(\mathrm{p}<0.05)$ were observed. HR before sleep was higher $(\mathrm{p}<0.01)$. Average HR while awake during sleep and light sleep stages significantly decreased $(\mathrm{p}<0.001)$. Such changes in all parameters were not observed after the BC group.

Conclusions Our study demonstrates that N-FDBC yields clinical benefits by improving sleep quality and HR response during sleep in healthy adults.

Sleep Med Res 2020;11(2):108-115

Key Words Breathing, Sleep, Heart rate. 
ority that a therapeutic program be developed that promotes sleep quality for those with sleep problems.

Sleep is a necessary physiological process that plays an important role in restorative functions that are essential for normal daytime [7]. During sleep, there are various stages of the sleep cycle including awake during sleep, the non-rapid eye movement stage (light sleep stage and deep sleep stage), and the rapid eye movement stage. For over a century many researchers have studied the sleep cycles, with findings indicating the light sleep stage is correlated with the physical restoration and the deep sleep stage with memory consolidation and immune system restoration [6]. Poor sleep quantity and quality have also been established as affecting the sleep cycle. In order to investigate the physiological changes during sleep, cardiorespiratory polysomnography (PSG) is regarded as the gold standard instrument in sleep medicine and sleep research [8]. However, PSG is time-consuming, performed in the laboratory, and expensive [9]. Recently, the smartwatch has received much attention as a tool for tracking sleep parameters. In clinical settings, the smartwatch is recommended as an adjunct in the assessment of sleep patterns in healthy adult populations [10,11].

To our knowledge, breathing exercises are widely acknowledged as a powerful intervention for cardiopulmonary systems with benefits including the lowering of blood pressure, heart rate (HR), and respiratory rate [12]. At present, various types of breathing exercises have been proven to improve cardiopulmonary parameters such as diaphragmatic breathing, the Pranayama breathing exercise, and deep breathing exercises [13]. Interestingly, a previous study has suggested that a breathing exercise with a rate of less than ten breaths per minute (bpm) compared with the usual rate of 12-20 bpm promotes parasympathetic nervous systems [12], via pulmonary stretch receptors stimulates the vagus nerve and the parasympathetic nervous system to reduce stress chemicals in the brain and facilitate a vascular relaxation response [14]. Furthermore, another study demonstrated that non-forced deep breathing in a supine position at a rate of 15,5 , and $3 \mathrm{bpm}$ resulted in a 1.5-fold increase in peak blood velocity in healthy young males [15]. Furthermore, forced deep breathing with a rate of 15,5 , and $3 \mathrm{bpm}$ significantly increased 2.0fold in peak blood velocity in healthy young males in the supine position [16]. Breathing exercises also have effects on the cardiovascular dynamic and autonomic nervous system.

From the above, it is clear that autonomic activity balance affects sleep quantity and quality. The pattern and rate of breathing also affect the autonomic activity balance, and a cardiovascular dynamic, breathing program with a rate lower than the usual may promote parasympathetic activity, consequently improving sleep drive. Breathing programs might improve sleep quantity and quality. Accordingly, we hypothesized that breathing cycle programs [forced deep breathing, non-forced deep breathing, and breathing control (BC)] at a rate lower than usual may improve sleep quantity, sleep quality, and HR during sleep.
Thus, this study aimed to investigate the effects of the forced deep breathing cycle (FDBC), non-forced deep breathing cycle (N-FDBC), and BC exercise program on sleep parameters and $\mathrm{HR}$ as measured by a smartwatch in healthy young adults.

\section{METHODS}

\section{Study Design and Ethical Considerations}

This study was a double-blinded randomized controlled trial. The purpose, benefits, and possible risks associated with the study were explained to the participants and informed consent was obtained, in accordance with the research ethics review committee for research involving human research participants, health sciences group, Chulalongkorn University (no. 029.1/63). The Thai Clinical Trials Registry (TCTR) identification number of this study is TCTR20200715004. All participants gave written informed consent before enrollment into the study.

\section{Study Participants and Characteristics}

All participants included in this study were apparently healthy without present illness or sleep problems. The inclusion criteria of the participants included healthy Thai males and females aged 18-24 years old without underlying disease and with normal sleep function. Normal sleep function was defined as a score on the Epworth Sleepiness Scale (ESS) of less than ten. The ESS is a short questionnaire, easy to understand and fill out, and its validity and repeatability for measurement for daytime sleepiness and sleep function. In general, ESS scores can be interpreted as follows: less than ten (normal), 11-12 (mild) 13-15 (moderate), and 16-24 (severe) excessive daytime sleepiness [17]. Participants with ESS more than ten and with a history of respiratory, cardiovascular, cardiometabolic, autoimmune conditions were excluded.

\section{Research Procedures}

The process for the research procedures is given in Fig. 1.

\section{Baseline assessments}

The 34 healthy participants (10 male and 24 female) were initially recruited to the study. All participants received a sleep hygiene education. Sleep hygiene comprises a variety of different methods that are required to have good sleep quality such as avoiding caffeine and strenuous workouts close to bedtime, preparing a good sleep environment, exposure to natural light, and a regular bedtime. Age, gender, smoking, ESS, and medical history were self-reported. Height and weight were measured, and body mass index (BMI) was calculated. Peripheral blood pressure and $\mathrm{HR}$ were measured by blood pressure monitor (Omrom, Japan) at rest on the left upper arm three times consecutively. The average values of the three times for the blood pressure and HR at rest were used. After recruitment, one of the partici- 


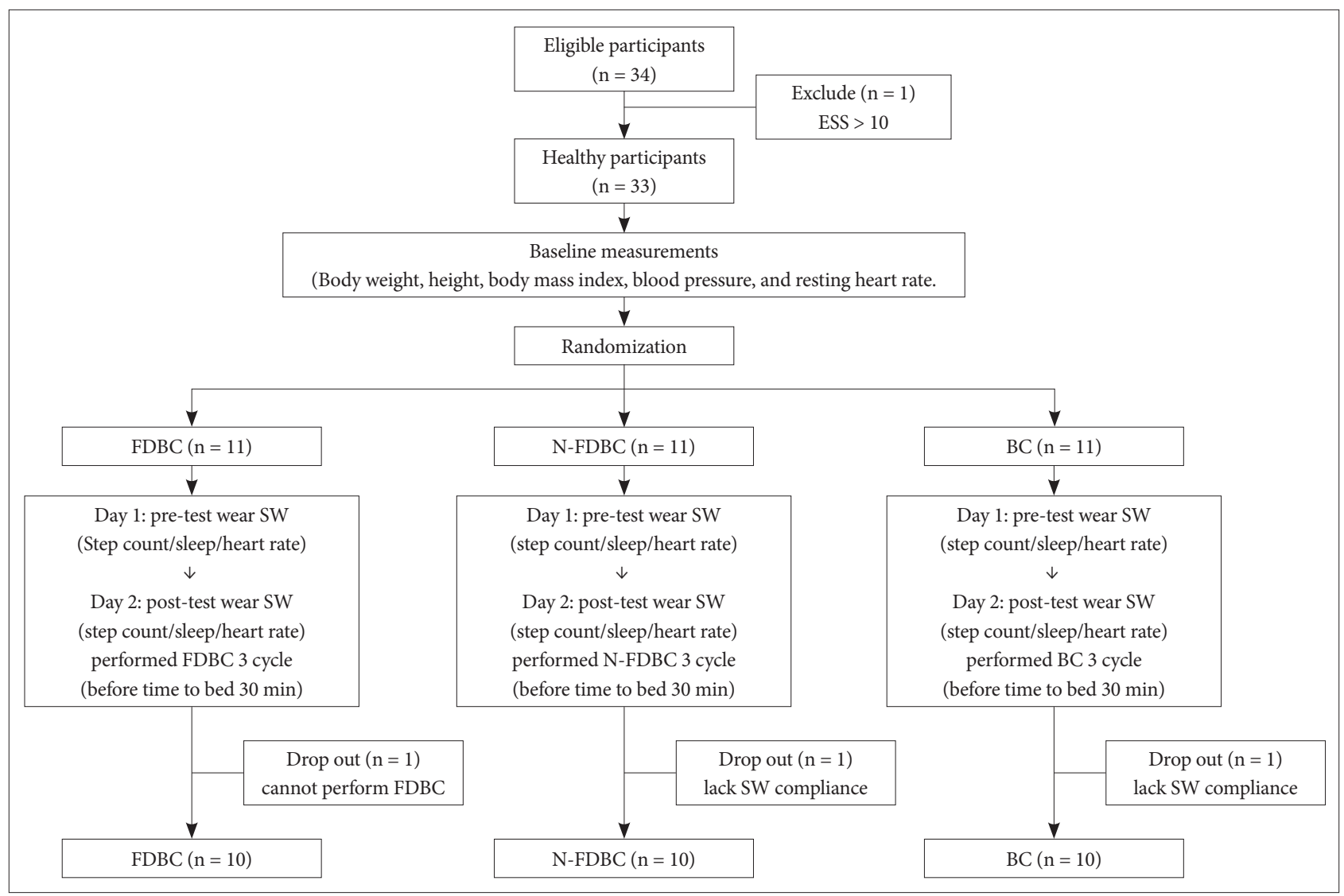

Fig. 1. The research procedure. ESS: Epworth Sleepiness Scale, SW: smartwatch, FDBC: forced deep breathing cycle, N-FDBC: nonforced deep breathing cycle, BC: breathing control.

pants was excluded from the study (ESS $>10$ scores). The remaining 33 participants were randomized and allocated equally into three groups through the simple block randomization method, namely, the FDBC group $(n=11)$, the N-FDBC group $(\mathrm{n}=11)$ and the BC group $(\mathrm{n}=11)$. After randomization, participants were instructed on the breathing cycle programs by the researcher. Participants were asked to wear a smartwatch all day (Apple Watch series 5, United States) for two consecutive days.

\section{Day 1 (Pre-test)}

Total step count, sleep, and HR parameters were recorded by smartwatch, participants were instructed to perform physical activity as per their usual day and regular sleep time.

\section{Day 2 (Post-test)}

Total step count, sleep, and HR parameters were recorded. At least 30 minutes before time to bed, each participant performed the breathing cycle programs. During performance of the breathing program, participants were advised to relax and reduce tension. All breathing cycle programs were performed for three cycles, with a rest by breathing with normal tidal volume for one minute after each cycle. One participant in FDBC dropped out as they could not perform the FDBC procedure, and the other one participant in N-FDBC and one participant in BC dropped out due to lack of smartwatch compliance during sleep. Ten participants per group completed the study as shown in Fig. 1.

\section{Breathing cycle programs}

FDBC

The FDBC was performed using the procedure described previously [16]. In brief, one cycle of FDBC comprises forced deep breaths with a rate of $15 \mathrm{bpm}$ for one minute, $5 \mathrm{bpm}$ for one minute, and $3 \mathrm{bpm}$ for one minute, respectively. One cycle lasted three minutes. For the rate of $15 \mathrm{bpm}$, participants inspired as fast as possible, held the breath for 2 seconds, then expired the air as fast as possible. For the rate of 5 and $3 \mathrm{bpm}$, participants perform similar maneuvers but held the breath for six and ten seconds, respectively. FDBC procedure video clip used to monitor breathing rates participants were instructed to perform three cycles of FDBC. Between each cycle, participants breathed as per their usual volume for one minute.

\section{$N-F D B C$}

The N-FDBC is similar to FDBC but participants were instructed to take a deep breath without force. Each cycle comprised three minutes of breathing. During the first minute, par- 
ticipants breathed at a rate of $15 \mathrm{bpm}$. During the second minute, participants breathed at a rate of $5 \mathrm{bpm}$. During the third minute, participants breathed at a rate of $3 \mathrm{bpm}$ consecutively. For the rate of $15 \mathrm{bpm}$, participants inspired slowly, held the breath for two seconds, then expired slowly. For the rate of 5 and $3 \mathrm{bpm}$, participants performed similar maneuvers but held the breath for six and ten seconds, respectively. N-FDBC procedure video clip used to monitor breathing rates. Participants were instructed to perform three cycles of N-FDBC. Between each cycle, participants breathed as per their usual volume for one minute.

\section{$B C$ exercise program}

Participants performed $\mathrm{BC}$ by breathing gently, using the least effort, focusing on the breathing muscles such as the diaphragm muscle, and put one hand on the chest and the other on the abdomen. Participants performed by slowly breathing in through the nose, with mouth closed, then breathing out through the mouth. Participants were instructed to perform three cycles of $\mathrm{BC}$, each cycle including BC for three minutes and breathing similar to their normal tidal volume for one minute.

\section{Outcome Measures}

\section{Total step count parameters}

The total step count was used to identify physical activity each day. The total step count was recorded for two consecutive days via Apple Watch series 5 (Apple Inc., Cupertino, CA, USA). Apple Watch tracked arm motion, measured by the built-in accelerometer, and global positioning system for estimating results including steps and distance. Participants were instructed to swings their arms naturally during two consecutive days.

\section{Sleep and HR parameters}

The sleep and HR parameters including time in bed, total sleep time, sleep latency, sleep quality, the percentage awake during sleep, the percentage rapid eye movement stage, the percentage light sleep stage, the percentage deep sleep stage, and average HR for each stage of sleep cycles were recorded for two consecutive nights. All parameters were assessed by Pillow Automatic Sleep Tracker (version 3.9.65; NeyBox Digital Ltd., Nicosia, Nicosia, Cyprus), Neybox Digital, Athens, Attica. The Pillow program analyzed changes in the sleep cycle and HR. To calculate sleep quality, the Pillow program takes into account body motions during sleep, as registered by the device's accelerometer and gyroscope, and also the noise level as recorded by the device's microphone. These data are processed by a scientifically backed algorithm to calculate the sleep quality index.

Previous studies suggested that smartwatch is recommended as an adjunct in the assessment of sleep patterns in healthy adult populations $[10,11]$. However, the validities and reliability of smartwatch (Apple Watch series 5) and Pillow Automatic Sleep Tracker (version 3.9.65) have not yet been reported. Be- fore data collection, we studied the correlation of sleep parameters and HR parameters measured by smartwatch (Apple Watch series 5) and Pillow Automatic Sleep Tracker (version 3.9.65) compare with PSG data analyzed by a medical specialist in ten healthy young adult subjects ( 5 males and 5 females). Significantly correlation between total sleep time $(\mathrm{r}=0.893, \mathrm{p}<0.05)$, sleep latency $(\mathrm{r}=0.935, \mathrm{p}<0.01)$, quality of sleep $(\mathrm{r}=0.843, \mathrm{p}<0.05)$, and average HR during sleep $(\mathrm{r}=0.812, \mathrm{p}<0.01)$ were observed. Thus, this study used smartwatch (Apple Watch series 5) and Pillow Automatic Sleep Tracker (version 3.9.65) to assessed sleep and HR parameters.

\section{Statistical Analyses}

Statistical analyses were performed using SPSS Statistics version 22 (IBM Corp., Armonk, NY, USA). Data were expressed as mean and standard deviation. Regarding normality, all of the data was analysed using the Shapiro-Wilk test, all data in this study were normal distribution. One-way analysis of variance (ANOVA) was used to assess any differences between demographic data, sleep and HR parameters in each group. Two-way ANOVA was used to compare sleep and HR parameters between the pre and post breath cycles programs in all FBDC, N-FBBC, and $\mathrm{BC}$ groups, and in case of a significant $\mathrm{F}$ test, Bonferroni' post-hoc was used to find individual differences. A value of $\mathrm{p}<$ 0.05 was considered statistically significant.

\section{RESULTS}

\section{Baseline Demographic Data}

All of the study participants were apparently healthy as evidenced by BMI, blood pressure, resting HR, and ESS within the normal range. The were no significant different anthropometrics, blood pressures, HR, and ESS between groups as shown in (Table 1).

\section{Total Step Count Parameter}

The total step count per day measured for the participants before the breathing cycle programs are presented in (Table 2). There were no significant differences between groups. This indicates that all participants had similar physical activity levels. Furthermore, physical activity levels each day were the same as evidenced by the total step count per day not being significant when compared within-group between the first day and second day in all groups.

\section{Sleep Quantity and Quality Parameters}

For the N-FDBC group, significant improvement in sleep quality was observed $(74.0 \pm 3.9 \%$ vs. $81.5 \pm 3.1 \%$, p $<0.001)$. Consequently, the percentage awake during sleep significantly decreased by $5 \%(\mathrm{p}<0.001)$. Total time in bed significantly increased $(428.7 \pm 28.7$ minutes vs. $445.7 \pm 27.8$ minutes, $\mathrm{p}<$ 
0.05). Total sleep time significantly increased (316.8 $\pm 28.5 \mathrm{~min}-$ utes vs. $364.0 \pm 32.2$ minutes, $\mathrm{p}<0.001$ ), reflecting a longer time in all light sleep and deep sleep stages. Furthermore, in the NFDBC group sleep latency significantly decreased by approximately $10 \mathrm{~min}(\mathrm{p}<0.001)$ comparing pre- and post-N-FDBC program.

On other hand, participants in FDBC found it difficult to get to sleep because it took a longer sleep latency after performing FDBC as shown by the significant increase in sleep latency of about 5.1 minutes post-FDBC compared to pre-FDBC $(\mathrm{p}<0.05)$. Surprisingly, even though FDBC made it harder to sleep, the trend for FDBC was the promotion of light sleep and deep sleep stage caused by a decrease in the percentage awake during sleep $(20.5 \pm 3.1 \%$ vs. $17.8 \pm 3.2 \%, \mathrm{p}<0.05)$.

There were no significant changes in sleep parameters after $\mathrm{BC}$ before the sleep was observed, as shown in (Table 2).

Table 1. Baseline demographic data

\begin{tabular}{lccc}
\hline \multicolumn{1}{c}{ Parameters } & $\begin{array}{c}\text { FDBC } \\
(\mathrm{n}=10)\end{array}$ & $\begin{array}{c}\text { N-FDBC } \\
(\mathrm{n}=10)\end{array}$ & $\begin{array}{c}\text { BC } \\
(\mathrm{n}=10)\end{array}$ \\
\hline Age (years) & $20.2 \pm 1.75$ & $20.7 \pm 2.06$ & $21 \pm 1.94$ \\
Gender $(\mathrm{M} / \mathrm{F})$ & $3 / 7$ & $3 / 7$ & $3 / 7$ \\
Weight $(\mathrm{kg})$ & $58.1 \pm 5.7$ & $61.0 \pm 5.9$ & $59.5 \pm 5.5$ \\
Hight $(\mathrm{cm})$ & $164.9 \pm 4.3$ & $165.1 \pm 5.0$ & $165.3 \pm 6.7$ \\
BMI $\left(\mathrm{kg} / \mathrm{m}^{2}\right)$ & $21.4 \pm 1.6$ & $22.4 \pm 1.3$ & $21.8 \pm 1.1$ \\
SBP $(\mathrm{mm} \mathrm{Hg})$ & $120.6 \pm 4.1$ & $121.5 \pm 3.1$ & $120.3 \pm 4.8$ \\
DAP $(\mathrm{mm} \mathrm{Hg})$ & $87.5 \pm 7.3$ & $86.1 \pm 5.7$ & $85.8 \pm 8.3$ \\
Resting heart rate (bpm) & $90.1 \pm 5.9$ & $90.8 \pm 5.7$ & $92.5 \pm 5.6$ \\
Epworth Sleepiness Scale & $1.9 \pm 0.9$ & $1.6 \pm 0.8$ & $1.3 \pm 0.5$ \\
\hline
\end{tabular}

Data are expressed as mean \pm SD.

FDBC: forced deep breathing cycles, N-FDBC: non-forced deep breathing cycles, BC: breathing control, $\mathrm{M}$ : male, F: female, BMI: body mass index, SBP: systolic blood pressure, DAP: diastolic blood pressure, bpm: beats per minute.

\section{HR Parameters during Sleep}

As regards HR during sleep, the N-FDBC program showed an improvement in cardiac response among participants during sleep, with significant decreases in HR before sleep (87.8 \pm 5.7 bpm vs. $82.2 \pm 4.9$ bpm, $\mathrm{p}<0.001$ ), maximum HR during sleep $(92.5 \pm 4.9$ bpm vs. $86.7 \pm 5.4 \mathrm{bpm}, \mathrm{p}<0.001)$, and minimum HR during sleep (72.3 \pm 4.2 bpm vs. $66.2 \pm 3.1$ bpm, $\mathrm{p}<$ $0.01)$. A significant decrease was found for the average $H R$ for all sleep stages except REM as shown by the average HR in the awake during sleep $87.4 \pm 5.8$ bpm vs. $80.0 \pm 4.5 \mathrm{bpm}, \mathrm{p}<0.001$ ), light sleep stage $(79.8 \pm 4.3 \mathrm{bpm}$ vs. $75.8 \pm 5.5 \mathrm{bpm}, \mathrm{p}<0.01)$, and deep sleep stage $(75.3 \pm 3.7 \mathrm{bpm}$ vs. $70.4 \pm 1.7 \mathrm{bpm}, \mathrm{p}<0.01)$.

On the other hand, a significant increase in HR before sleep $(87.1 \pm 5.9$ bpm vs. $92.2 \pm 6.1 \mathrm{bpm}, \mathrm{p}<0.01)$ were observed following the FDBC program. However, average HR during awake during sleep $(83.8 \pm 5.8$ bpm vs. $78.0 \pm 2.7 \mathrm{bpm}, \mathrm{p}<$ $0.001)$ and light sleep stage $(75.4 \pm 6.3 \mathrm{bpm}$ vs $72.8 \pm 3.6 \mathrm{bpm}$, $\mathrm{p}<0.001)$ significantly decreased after the FDBC program.

No changes in HR parameters were found in the $\mathrm{BC}$ group as shown in (Table 3).

\section{DISCUSSION}

Our study is the first study to measure the effect of FDBC and $\mathrm{N}-\mathrm{FDBC}$ programs on sleep and HR parameters during sleep. The present study demonstrates the effect of the breathing cycle program on sleep and HR parameters. The main findings of this study were: 1) the breathing cycle program affected sleep parameters as evidenced by the N-FDBC program, significant improvement in time in bed, time asleep sleep latency, quality of sleep, and percentage awake during sleep. For the FDBC program, there were significant changes in sleep latency, and the percentage awake during sleep, 2) the breathing cycle program also affected HR parameters as found by the significant decrease

Table 2. Effects of breathing cycle program on sleep parameters

\begin{tabular}{|c|c|c|c|c|c|c|}
\hline \multirow{2}{*}{ Sleep parameters } & \multicolumn{2}{|c|}{ FDBC } & \multicolumn{2}{|c|}{ N-FDBC } & \multicolumn{2}{|c|}{$\mathrm{BC}$} \\
\hline & Pre & Post & Pre & Post & Pre & Post \\
\hline Total step count per day (steps) & $10552 \pm 933$ & $10481 \pm 311$ & $10929 \pm 689$ & $10849.2 \pm 509$ & $10722.1 \pm 1248$ & $10764.1 \pm 632$ \\
\hline Time in bed (minutes) & $437.6 \pm 40.1$ & $443.9 \pm 29.5$ & $428.7 \pm 28.7$ & $445.7 \pm 27.8^{*}$ & $454.2 \pm 31.6$ & $450.6 \pm 20.7$ \\
\hline Time asleep (minutes) & $327.7 \pm 30.4$ & $339.9 \pm 32.8$ & $316.8 \pm 28.5$ & $364.0 \pm 32.2^{\dagger}$ & $326.6 \pm 31.7$ & $320.3 \pm 18.8$ \\
\hline Sleep latency (minutes) & $20.1 \pm 5.6$ & $25.2 \pm 3.4^{*}$ & $20.2 \pm 6.9$ & $10.7 \pm 2.2^{\dagger}$ & $19.3 \pm 4.3$ & $22.0 \pm 3.1$ \\
\hline Quality of sleep (\%) & $74.8 \pm 3.4$ & $76.5 \pm 3.2$ & $74.0 \pm 3.9$ & $81.5 \pm 3.1^{\dagger}$ & $71.8 \pm 5.8$ & $71.4 \pm 4.9$ \\
\hline Awake during sleep (\%) & $20.5 \pm 3.1$ & $17.8 \pm 3.2^{*}$ & $21.4 \pm 3.6$ & $16.0 \pm 3.2^{\dagger}$ & $24.5 \pm 6.2$ & $23.9 \pm 5.2$ \\
\hline Light sleep (\%) & $40.3 \pm 6.4$ & $39.1 \pm 6.1$ & $37.5 \pm 9.3$ & $40.4 \pm 3.9$ & $41.0 \pm 6.7$ & $41.2 \pm 2.8$ \\
\hline Deep sleep (\%) & $29.6 \pm 8.2$ & $32.9 \pm 6.9$ & $28.0 \pm 7.6$ & $32.4 \pm 5.4$ & $23.6 \pm 8.2$ & $24.3 \pm 4.7$ \\
\hline REM (\%) & $9.6 \pm 6.3$ & $10.2 \pm 6.3$ & $13.1 \pm 9.1$ & $11.2 \pm 7.2$ & $10.9 \pm 6.6$ & $10.6 \pm 5.0$ \\
\hline
\end{tabular}

Data are expressed as mean \pm SD.

${ }^{*} \mathrm{p}<0.05 .{ }^{\dagger} \mathrm{p}<0.001$ compared between pre and post within group.

FDBC: forced deep breathing cycles, N-FDBC: non-forced deep breathing cycles, BC: breathing control, REM: rapid eye movement. 
Table 3. Effects of breathing cycle program on HR during sleep

\begin{tabular}{|c|c|c|c|c|c|c|}
\hline \multirow{2}{*}{ HR parameters } & \multicolumn{2}{|c|}{ FDBC } & \multicolumn{2}{|c|}{ N-FDBC } & \multicolumn{2}{|c|}{$\mathrm{BC}$} \\
\hline & Pre & Post & Pre & Post & Pre & Post \\
\hline HR before sleep (bpm) & $87.1 \pm 5.9$ & $92.2 \pm 6.1^{*}$ & $87.8 \pm 5.7$ & $82.2 \pm 4.9^{\dagger}$ & $89.5 \pm 5.6$ & $89.2 \pm 6.8$ \\
\hline Max HR during sleep (bpm) & $90.3 \pm 5.2$ & $90.8 \pm 5.1$ & $92.5 \pm 4.9$ & $86.7 \pm 5.4^{\dagger}$ & $91.7 \pm 3.2$ & $90.8 \pm 4.2$ \\
\hline Min HR during sleep (bpm) & $70.8 \pm 5.8$ & $69.6 \pm 3.2$ & $72.3 \pm 4.2$ & $66.2 \pm 3.1^{*}$ & $72.7 \pm 4.1$ & $72.1 \pm 3.8$ \\
\hline Average awake HR (bpm) & $83.8 \pm 5.8$ & $78.0 \pm 2.7^{\dagger}$ & $87.4 \pm 5.8$ & $80.0 \pm 4.5^{\dagger}$ & $85.3 \pm 4.3$ & $83.8 \pm 3.3$ \\
\hline Average light sleep HR (bpm) & $79.9 \pm 5.3$ & $74.4 \pm 3.0^{\dagger}$ & $79.8 \pm 4.3$ & $75.8 \pm 5.5^{*}$ & $79.4 \pm 3.6$ & $77.5 \pm 1.6$ \\
\hline Average deep sleep HR (bpm) & $75.4 \pm 6.3$ & $72.8 \pm 3.6$ & $75.3 \pm 3.7$ & $70.4 \pm 1.7^{*}$ & $75.5 \pm 3.7$ & $73.7 \pm 4.1$ \\
\hline Average REM HR (bpm) & $83.0 \pm 5.3$ & $80.4 \pm 3.7$ & $81.7 \pm 2.8$ & $82.9 \pm 3.7$ & $80.8 \pm 5.4$ & $81.6 \pm 2.8$ \\
\hline
\end{tabular}

Data are expressed as mean \pm SD.

${ }^{*} \mathrm{p}<0.01 .{ }^{\dagger} \mathrm{p}<0.001$ compared between pre and post within group.

FDBC: forced deep breathing cycles, N-FDBC: non-forced deep breathing cycles, BC: breathing control, HR: heart rate, bpm: beats per minute, REM: rapid eye movement.

in HR before sleep, maximum HR during sleep, minimum HR during sleep, and average HR during all sleep stages except the REM stage were observed after the N-FDBC program. Moreover, after the FDBC program significant changes in HR before sleep, HR during awake during sleep and light sleep stage were found.

This study recorded the total step count per day. There were no significant differences at baseline between groups. The total step count on the first and second days showed no significant differences compared within group. Therefore, this result can eliminate the effect of physical activity on the sleep function.

\section{Breathing Cycle Program on Sleep Parameters}

To the extent of our knowledge, the relationship between sleep quality and the parasympathetic nervous system, which is a physiological phenomenon that is a parasympathetic drive, promotes sleep [6]. From the literature review, breathing patterns are also associated with autonomic nervous systems via respiratory sinus arrhythmia due to changes in the cardiovagal system, resulting in autonomic reactivity [13]. In this study, N-FDBC program showed significant improvement in time in bed, time asleep, sleep latency, quality of sleep, and the percentage awake during sleep. On the other hand, the FDBC program showed significant changes in sleep latency, and the percentage awake during sleep. This discrepancy is probably due to differences in pattern and rate that may also play a role in the parasympathetic response as a lower ratio had a positive effect on HR variability only with slow respiratory rates but not fast respiratory rates [9].

In the present study, for the FDBC program sleep latency increased by about 5.1 minutes. During respiration in the FDBC program, participants had to take forced deep breaths for both inspiration and expiration, and hold the breaths for 2, 6, and 10 seconds. Previous studies reported that a breathing pattern with a hold period assists increases the venous return of the blood. This maneuver is also termed a respiratory pump [16]. Regulation of the venous return is influenced by both the sympathetic and parasympathetic nervous systems simultaneously [5]. FDBC involved a holding period of breath, which affects venous return and autonomic balance. Another study found that a forced breathing program stimulates vagal tone to modulate the sinoatrial node, increases HR oscillation, and increases sympathetic activity [6]. Increasing the sleep latency after performing FDBC program may be explained by the fact that the FDBC pattern involved a holding period of breath and participants had to force their breathing during performance of the FDBC program. The FDBC may increase the venous return, activating the sympathetic nervous system leading to increased sympathetic activity, and resulting in delayed sleep latency. However, there has been no study concerning about the FDBC on sleep parameters, the decreased percentage of awake during found in this study may be reflect a change of autonomic balance. Furthermore, in the future study, HR variability (represent autonomic activity) during perform FDBC and other pattern of breathing should be measured to confirm their effect on autonomic nervous system.

Another observation made in our study was the improved sleep parameter after N-FDBC compared to baseline. Several studies have investigated the effect of slow deep breathing [13,18-20]. A previous study found that slow deep breathing is a simple maneuver that stimulates the parasympathetic system, thereby resulting in increased parasympathetic activity [18]. Moreover, another study researched the effect of slow breathing on the R-R interval, concluding that breathing patterns were associated with the cardiovagal system [19]. It was also observed that slow deep breathing at a rate of less than $12 \mathrm{bpm}$ increased the high frequency (HF) component of HR variability [20], and significantly increased in HF during deep breathing in the supine posture at 10-12 bpm $(0.17-0.20 \mathrm{~Hz})$ [13]. This indicated slow deep breathing stimulates the primary parasympathetic system. The improved sleep parameters in our study could be due to the slow deep breathing pattern in the N-FDBC program. The N-FDBC program may affect the $\mathrm{R}-\mathrm{R}$ interval, leading to activation of the cardiovagal system, which primarily stimulates parasympathetic activity, resulting in improved sleep quantity and quality as 
observed in our study.

\section{Breathing Cycle Program on HR Parameters during Sleep}

According to the results of this study, FDBC and N-FDBC decrease HR during sleep. However, only FDBC showed a significant increase in HR before sleep. Several studies have reported changes in HR after breathing exercise [6,12-14]. Most of the studies revealed a significant decrease in HR $[6,12,13]$, or no change [14]. Changes in HR are influenced by both the sympathetic and parasympathetic nervous systems [5]. A previous study asserted that the force inspiration and expiration breathing pattern stimulated sympathetic activity $[5,6]$, and the breathing pattern with a hold period increases the venous return of blood [16], leading to an increased HR due to the physiological changes for maintaining cardiovascular homeostasis. The FDBC program had both forced deep breathing and period of holding the breath. The reason FDBC increases the HR may be due to the FDBC program possibly stimulating the vagal tone by modulating the sinoatrial node and increasing sympathetic activity, leading to an increased HR caused by the respiration pattern $[5,16]$. Another possible explanation for the increased $\mathrm{HR}$ before sleep observed in the present study, maybe the FDBC increased the venous return to the heart via the respiratory pump [16], and an increasing HR response might be observed for controlling cardiovascular homeostasis. The results of our study indicate increased sleep latency and increased HR before sleep. However, data from previous studies support the potential of FDBC program may increase sympathetic activity via various mechanisms $[5,16]$. In this study, we observed decreased HR during the awake sleep stage and light sleep stage, and there have been no reports on the HR response during sleep after performing FDBC program. Therefore, we need to examine the effects of FDBC and other respiratory patterns on HR variability, in future studies.

Another noteworthy observation made in our study was the significant improvements in HR before sleep, maximum HR during sleep, minimum HR during sleep, and average HR during all sleep stages except the REM stage following the N-FDBC program. The N-FDBC program had a slow deep breath pattern with a rate slower than the usual respiratory rate. A previous study suggested that slow deep breaths without being forced promote primary parasympathetic activity [13]. Our finding is in agreement with a previous study that deep breathing at a rate of $10^{-}$ 12 bpm increased parasympathetic activity [13,18-20]. Therefore, it is most likely that N-FDBC improves sleep and HR parameters through the pattern of breathing, with the slow deep breathing without being forced promoting parasympathetic activity. Further study should investigate changes in HR variability after the N-FDBC program.

\section{Clinical Implications}

The current findings revealed that the breathing cycles pro- gram influenced sleep quantity, sleep quality, and HR response during sleep. Our findings suggest clinicians should use a breathing cycle program to promote restoration. The breathing cycles program can contribute to promoting sleep quantity and quality, leading to improved restoration function during sleep, resulting in minimizing the risk of mental health problems, cardiovascular diseases, cardiometabolic diseases, neurological diseases, and other diseases. Consequently, people experiencing sleep problems should perform a breathing cycles program as these programs may ameliorate the sleep difficulties.

\section{Limitations}

This study is the first to examine the effect of the FDBC and N-FDBC program on sleep and HR parameters. Breathing cycle programs have the advantage of promoting sleep function and HR during sleep without the need for any special devices, but they are not without some limitations. Firstly, although the FDBC and N-FDBC programs change sleep and HR parameters immediately, it remains unclear if this effect can be sustained throughout the night. Longitudinal research is needed to evaluate the effect of deep breathing without force on cardiac-vagal activity, sleep function, and HR response during sleep. Second, this study used the smartwatch (Apple Watch series 5) to tracking movement and HR and used the Pillow application (version 3.9.65) for analysis the sleep parameters the results reporting as the average sleep function and HR throughout the night. Further study should determine sleep function and HR via PSG (gold standard tools for sleep assessment).

\section{Conclusion}

In summary, our study demonstrates the benefit of breathing cycle programs on sleep function and HR during sleep. The NFDBC showed significant improvement in sleep function, as evidenced by increased sleep quality and decreased HR during sleep.

\section{Acknowledgments}

This study received grant for development of new faculty staff, Ratchadaphisaksomphot Endowment Fund, Chulalongkorn University.

\section{Conflicts of Interest}

The authors have no financial conflicts of interest

\section{Authors' Contribution}

All authors designed the study (conceptualization), Jirawat Wattanapanyawech was responsible for data collection and statistical analyses. Premtip Thaveeratitham revised the content of the paper. All authors contributed to the preparation of the article and approved the final submitted version.

\section{REFERENCES}

1. Ohayon MM. Epidemiological overview of sleep disorders in the general population. Sleep Med Res 2011;2:1-9.

2. Luckhaupt SE, Tak S, Calvert GM. The prevalence of short sleep duration by industry and occupation in the National Health Interview Sur- 
vey. Sleep 2010;33:149-59.

3. Lin M, Ye M, Peng J, Yin K, Wang Z. The employees' sleep quality: a perspective of organizational behavior. Advances in Psychological Science 2018;26:1096-110.

4. Suraev AS, Marshall NS, Vandrey R, McCartney D, Benson MJ, McGregor IS, et al. Cannabinoid therapies in the management of sleep disorders: a systematic review of preclinical and clinical studies. Sleep Med Rev 2020;53:101339.

5. Lehrer PM, Vaschillo E, Vaschillo B, Lu SE, Eckberg DL, Edelberg R, et al. Heart rate variability biofeedback increases baroreflex gain and peak expiratory flow. Psychosom Med 2003;65:796-805.

6. Allen TD, Kiburz KM. Trait mindfulness and work-family balance among working parents: the mediating effects of vitality and sleep quality. J Vocat Behav 2012;80:372-9.

7. Liu K, Yin T, Shen Q. Relationships between sleep quality, mindfulness and work-family conflict in Chinese nurses: a cross-sectional study. Appl Nurs Res 2020;55:151250.

8. Wuttiumporn K, Khrisanapant W, Pasurivong O, Boonsawat W, Intarapoka B. Cardiovascular performance following continuous positive airway pressure in patients with severe obstructive sleep apnea. Sleep Hypn 2018;20:174-83.

9. Zinkhan M, Berger K, Hense S, Nagel M, Obst A, Koch B, et al. Agreement of different methods for assessing sleep characteristics: a comparison of two actigraphs, wrist and hip placement, and self-report with polysomnography. Sleep Med 2014;15:1107-14.

10. Kushida CA, Littner MR, Morgenthaler T, Alessi CA, Bailey D, Coleman J Jr, et al. Practice parameters for the indications for polysomnography and related procedures: an update for 2005. Sleep 2005;28:499521.

11. Morgenthaler T, Alessi C, Friedman L, Owens J, Kapur V, Boehlecke B, et al. Practice parameters for the use of actigraphy in the assessment of sleep and sleep disorders: an update for 2007. Sleep 2007;30:519-29.

12. Brenner J, LeBlang S, Lizotte-Waniewski M, Schmidt B, Espinosa PS,
DeMets DL, et al. Mindfulness with paced breathing reduces blood pressure. Med Hypotheses 2020;142:109780.

13. Liu YW, Tzeng NS, Yeh CB, Kuo TBJ, Huang SY, Chang CC, et al. Reduced cardiac autonomic response to deep breathing: a heritable vulnerability trait in patients with schizophrenia and their healthy firstdegree relatives. Psychiatry Res 2016;243:335-41.

14. Herrero JL, Khuvis S, Yeagle E, Cerf M, Mehta AD. Breathing above the brain stem: volitional control and attentional modulation in humans. $J$ Neurophysiol 2018;119:145-59.

15. Stein PD, Yaekoub AY, Ahsan ST, Matta F, Lala MM, Mirza B, et al. Ankle exercise and venous blood velocity. Thromb Haemost 2009;101:1100-3.

16. Nakanishi K, Takahira N, Sakamoto M, Yamaoka-Tojo M, Katagiri M, Kitagawa J. Effects of forced deep breathing on blood flow velocity in the femoral vein: developing a new physical prophylaxis for deep vein thrombosis in patients with plaster cast immobilization of the lower limb. Thromb Res 2018;162:53-9.

17. Gander PH, Marshall NS, Harris R, Reid P. The Epworth Sleepiness Scale: influence of age, ethnicity, and socioeconomic deprivation. Epworth Sleepiness scores of adults in New Zealand. Sleep 2005;28:24953.

18. Przybylska-Felus M, Furgala A, Zwolinska-Wcislo M, Mazur M, Widera A, Thor P, et al. Disturbances of autonomic nervous system activity and diminished response to stress in patients with celiac disease. J Physiol Pharmacol 2014;65:833-41.

19. Pitzalis MV, Mastropasqua F, Massari F, Passantino A, Colombo R, Mannarini A, et al. Effect of respiratory rate on the relationships between RR interval and systolic blood pressure fluctuations: a frequency-dependent phenomenon. Cardiovasc Res 1998;38:332-9.

20. Dekker JM, Crow RS, Folsom AR, Hannan PJ, Liao D, Swenne CA, et al. Low heart rate variability in a 2-minute rhythm strip predicts risk of coronary heart disease and mortality from several causes: the ARIC Study. Atherosclerosis Risk In Communities. Circulation 2000;102:123944. 